Gen Relativ Gravit manuscript No.

(will be inserted by the editor)

Burin Gumjudpai

\title{
Scalar field exact solutions for non-flat FLRW cosmology: A technique from non-linear Schrödinger-type formulation
}

Received: date / Accepted: date

\begin{abstract}
We report a method of solving for canonical scalar field exact solution in a non-flat FLRW universe with barotropic fluid using non-linear Schrödinger (NLS)-type formulation in comparison to the method in the standard Friedmann framework. We consider phantom and non-phantom scalar field cases with exponential and power-law accelerating expansion. Analysis on effective equation of state to both cases of expansion is also performed. We speculate and comment on some advantage and disadvantage of using the NLS formulation in solving for the exact solution.
\end{abstract}

Keywords Scalar field cosmology · Non-linear Schrödinger equation · Power-law expansion · Exponential expansion

PACS $98.80 . \mathrm{Cq}$

\section{Introduction}

In the past decade, it has been observed that universe is now in acceleration phase [1,2,3, , w wile inflationary scenario of the early universe 5 is strongly confirmed by cosmic microwave background data 6,7, 8, 9. In both circumstances, the universe experiences accelerating expansion which can be attained by exploiting some dynamical scalar field with time-dependent equation of state coefficient $w_{\phi}(t)<-1 / 3$, or a cosmological constant with $w_{\Lambda}=-1$ [10]. It was further suggested that the scalar field could have phantom behavior with $w_{\phi}<-1$ as one considers kinetic energy term in its Lagrangian density to be negative [11,12. Strong supports to the phantom idea are from observations previously, e.g. combined cosmic microwave background, large scale structure survey and supernovae type Ia without assuming flat universe yields $w_{\phi, 0}=-1.06_{-0.08}^{+0.13}[$ [] while using supernovae data alone assuming flat universe, $w_{\phi, 0}=-1.07 \pm 0.09$ 13. The subscript 0 denotes the value at present. Moreover, most recent WMAP five-year result [7,8, combined with Baryon Acoustic Oscillation (BAO) of large scale structure survey from SDSS and 2dFGRS 14 and type Ia supernovae data from HST [3], SNLS [4] and ESSENCE [13] assuming dynamical $w$ with flat universe yields $-1.38<w_{\phi, 0}<-0.86$ at $95 \%$ confident level and $w_{\phi, 0}=-1.12 \pm 0.13$ at $68 \%$ confident level 9. With additional BBN constraint of limit of expansion rate [15] 16, $-1.32<w_{\phi, 0}<-0.86$ at $95 \%$ confident level and $w_{\phi, 0}=-1.09 \pm 0.12$

This work is supported by a TRF-CHE Research Career Development Grant of the Thailand Research Fund and the Naresuan Faculty of Science Research Scheme.

Burin Gumjudpai

Fundamental Physics \& Cosmology Research Unit, The Tah Poe Academia Institute (TPTP)

Department of Physics, Naresuan University, Phitsanulok 65000, Thailand

and

School of Physics, Institute of Science, Suranaree University of Technology

111 University Avenue, Nakhon Ratchasima 30000, Thailand

E-mail: buring@nu.ac.th 
at $68 \%$ confident level. This suggests that phantom field has firmed status in cosmology. However, phantom field does result in unwanted Big Rip singularity in a flat FLRW universe [17. This raises up many attempts to avoid the singularity based on both phenomenological and fundamental inspirations [18.

Recently, there are a few proposals for mathematical alternatives to the conventional Friedmann formulation of canonical scalar field cosmology, such as non-linear Ermakov-Pinney equation [19,20. Moreover, a non-Ermakov-Pinney equation for the same system was also proposed in form of a nonlinear Schrödinger-type equation (hereafter-NLS) and it was found that solutions of the NLS-type equation are correspondent to solutions of the generalized Ermakov-Pinney equation [20, 21]. Conclusion of how to relate NLS quantities to quantities of standard Friedmann formulation is shown in [23] which gives extension to phantom field case. It also shows that the NLS wave function is in general nonnormalizable. Expressing cosmological quantities in form of NLS quantities may suggest an alternative way of solving problems in scalar field cosmology. In such method, presumed knowledge of scale factor function with time $a(t)$ must be given first and later one can evaluate NLS potential based on $a(t)$ assumed. Here we attempt to solve for scalar field exact solution within the NLS framework in various cases with flat and non-flat spatial geometries. We compare the results to the solutions obtained in standard Friedmann formulation. Exponential expansion $a \sim \exp (t / \tau)$ and power-law expansion $a \sim t^{q}$ are assumed where $\tau$ are finite characteristic time and $q$ is a positive constant.

This article is organized as follow. We express our cosmological system in Sec. 2 before introducing the NLS formulation in Sec. 3. Afterward, we consider each model of expansion separately. Exponential expansion is presented in Sec.4 where we obtain exact solution from effective equation of state and later we solve for exact solution from Friedmann system. Afterward, in Sec. 5, we consider NLS formulation for the exponential expansion and solve for exact solutions in NLS framework. We analyze effective equation of state for the exponential expansion case in Sec. 6. When considering power-law expansion, we work and organize the contents in the same spirit and order as in the previous sections. Beginning from Sec. 7, 8, and 9. Finally, conclusion is made in Sec. 10.

\section{Cosmological System}

To be realistic, two perfect fluids are considered in our system: barotropic fluid and scalar field fluid. The perfect barotropic fluid pressure $p_{\gamma}$ and density $\rho_{\gamma}$ obey an equation of state, $p_{\gamma}=(\gamma-1) \rho_{\gamma}=w_{\gamma} \rho_{\gamma}$ while for scalar field, $p_{\phi}=w_{\phi} \rho_{\phi}$. Total density and total pressure are $\rho_{\text {tot }}=\rho_{\gamma}+\rho_{\phi}$ and $p_{\text {tot }}=p_{\gamma}+p_{\phi}$. The effective equation of state is weighed-value of these two components,

$$
w_{\mathrm{eff}}=\frac{\rho_{\phi} w_{\phi}+\rho_{\gamma} w_{\gamma}}{\rho_{\mathrm{tot}}} .
$$

For the barotropic fluid, its equation of state coefficient $w_{\gamma}$ is written in term of $n$. We set $w_{\gamma} \equiv(n-3) / 3$ so that $n=3\left(1+w_{\gamma}\right)=3 \gamma$, hence $w_{\gamma}=-1$ corresponds to $n=0, w_{\gamma}=-1 / 3$ to $n=2, w_{\gamma}=0$ to $n=3, w_{\gamma}=1 / 3$ to $n=4$, and $w_{\gamma}=1$ to $n=6$. The conservation equation is therefore

$$
\dot{\rho}_{\gamma}=-n H \rho_{\gamma}
$$

with solution obtained directly,

$$
\rho_{\gamma}=\frac{D}{a^{n}}
$$

therefore $p_{\gamma}=[(n-3) / 3]\left(D / a^{n}\right)$, where $a$ is scale factor, the dot denotes time derivative, $H=\dot{a} / a$ is Hubble parameter and $D \geq 0$ is a proportional constant. The scalar field considered here is minimally coupling to gravity with Lagrangian density, $\mathcal{L}=(1 / 2) \epsilon \dot{\phi}^{2}-V(\phi)$, where $\epsilon=1$ for non-phantom case and -1 for phantom case. Density and pressure of the field are given as

$$
\rho_{\phi}=\frac{1}{2} \epsilon \dot{\phi}^{2}+V(\phi), \quad p_{\phi}=\frac{1}{2} \epsilon \dot{\phi}^{2}-V(\phi)
$$

\footnotetext{
1 Considering Bianchi I scalar field cosmology, one can also construct a corresponding linear Schrödinger-type equation by redefining cosmological quantities 22 .
} 
therefore

$$
w_{\phi}=\frac{p_{\phi}}{\rho_{\phi}}=\frac{\epsilon \dot{\phi}^{2}-2 V(\phi)}{\epsilon \dot{\phi}^{2}+2 V(\phi)} .
$$

The field obeys conservation equation

$$
\epsilon[\ddot{\phi}+3 H \dot{\phi}]+\frac{\mathrm{d} V}{\mathrm{~d} \phi}=0 .
$$

Considering Friedmann-Lemaître-Robertson-Walker (FLRW) universe, the Friedmann equation and acceleration equation are

$$
\begin{aligned}
H^{2} & =\frac{\kappa^{2}}{3} \rho_{\text {tot }}-\frac{k}{a^{2}}, \\
\frac{\ddot{a}}{a} & =-\frac{\kappa^{2}}{6} \rho_{\mathrm{tot}}\left(1+3 w_{\mathrm{eff}}\right)
\end{aligned}
$$

where $\kappa^{2} \equiv 8 \pi G=1 / M_{\mathrm{P}}^{2}, G$ is Newton's gravitational constant, $M_{\mathrm{P}}$ is reduced Planck mass, $k$ is spatial curvature. Using Eqs. (3), (4), (6) and (7), it is straightforward to show that

$$
\begin{aligned}
\epsilon \dot{\phi}(t)^{2} & =-\frac{2}{\kappa^{2}}\left[\dot{H}-\frac{k}{a^{2}}\right]-\frac{n D}{3 a^{n}}, \\
V(\phi) & =\frac{3}{\kappa^{2}}\left[H^{2}+\frac{\dot{H}}{3}+\frac{2 k}{3 a^{2}}\right]+\left(\frac{n-6}{6}\right) \frac{D}{a^{n}} .
\end{aligned}
$$

\section{Non-linear Schrödinger-type formulation}

Correspondence between non-linear Schrödinger formulation for canonical scalar field cosmology with barotropic fluid was shown in 21 and was concluded recently in 23. In the Schrödinger formulation, wave function $u(x)$ is related to scale factor in cosmology as

$$
u(x) \equiv a(t)^{-n / 2},
$$

and Schrödinger total energy $E$ and Schrödinger potential $P(x)$ are linked to cosmology as

$$
\begin{aligned}
E & \equiv-\frac{\kappa^{2} n^{2}}{12} D, \\
P(x) & \equiv \frac{\kappa^{2} n}{4} a(t)^{n} \epsilon \dot{\phi}(t)^{2} .
\end{aligned}
$$

These quantities satisfy non-linear Schrödinger-type equation:

$$
\frac{\mathrm{d}^{2}}{\mathrm{~d} x^{2}} u(x)+[E-P(x)] u(x)=-\frac{n k}{2} u(x)^{(4-n) / n} .
$$

The mapping from $t$ to $x$ is via $x=\sigma(t)$, such that

$$
\begin{aligned}
& \dot{x}(t)=u(x), \\
& \phi(t)=\psi(x) .
\end{aligned}
$$

We comment that the relation $\psi^{\prime}(x)^{2}=\left(4 / n \kappa^{2}\right) P(x)$ in Ref. 21] does not include phantom field case. Modification is made in recent work [23] so that the solution includes the phantom field case, therefore

$$
\psi(x)= \pm \frac{2}{\kappa \sqrt{n}} \int \sqrt{\frac{P(x)}{\epsilon}} \mathrm{d} x .
$$

If $P(x) \neq 0$ and $n \neq 0$. There exists an inverse function of $\psi(x)$ as $\psi^{-1}(x)$. Therefore $x(t)=\psi^{-1} \circ \phi(t)$ and the scalar field potential, $V \circ \sigma^{-1}(x)$ can be expressed as function of time,

$$
V(t)=\frac{12}{\kappa^{2} n^{2}}\left(\frac{\mathrm{d} u}{\mathrm{~d} x}\right)^{2}-\frac{2 u^{2}}{\kappa^{2} n} P(x)+\frac{12 u^{2}}{\kappa^{2} n^{2}} E+\frac{3 k u^{4 / n}}{\kappa^{2}} .
$$




\section{Exponential expansion}

4.1 Solution solved from effective equation of state for $k=0$ case

Exponential expansion reads

$$
a(t)=\exp (t / \tau),
$$

where $\tau$ is a positive constant. Flat universe undergoes exponential expansion only when $w_{\text {eff }}=-1$. The effective equation of state, (Eq. (1)) with Eqs. (4) and (5) can therefore be written a:

$$
\epsilon \dot{\phi}^{2}=-\frac{n}{3} \rho_{\gamma},
$$

which can be integrated directly, using Eq. (3), to

$$
\phi(t)= \pm 2 \tau \sqrt{\frac{D}{3 n}} e^{-n t / 2 \tau}+\phi_{0} .
$$

The solution above is obtained when assuming phantom scalar field, i.e. $\epsilon=-1$. If the scalar field is not phantom, the solution is imaginary.

\subsection{Solution solved from Friedmann formulation}

Another way to find the exact solution is to use Eq. (19), in Eq. (9). Therefore

$$
\epsilon \dot{\phi}(t)^{2}=\frac{2 k}{\kappa^{2}} e^{-2 t / \tau}-\frac{n D}{3} e^{-n t / \tau},
$$

which gives an integration:

$$
\phi(t)= \pm \int \sqrt{\frac{1}{\epsilon}\left(\frac{2 k}{\kappa^{2}} e^{-2 t / \tau}-\frac{n D}{3} e^{-n t / \tau}\right)} \mathrm{d} t .
$$

\subsubsection{Simplest case}

In the case of $k=0$ and $D=0$, the integration yields a constant $\phi_{0}$. Eq. (1) becomes $w_{\phi}=-1$. This is a cosmological constant as seen in simplest model of exponential expansion. When assuming only $k=0$ and $\epsilon=-1$ but with $D \neq 0$, the solution of Eq. (23) is the same as the Eq. (21) previously. For a scalar field domination in a non-flat universe $(D=0, k \neq 0)$, the solution is

$$
\phi(t)= \pm \frac{\tau}{\kappa} \sqrt{\frac{2 k}{\epsilon}} e^{-t / \tau}+\phi_{0} .
$$

where $k$ and $\epsilon$ must have the same sign, otherwise the solution is imaginary.

\subsubsection{The case of non-zero $k$ and non-zero $D$}

When $k$ and $D$ are both not negligible. Performing integration to the Eq. (23) is more complicated and could be impossible unless assumption of barotropic fluid type. When assuming a particular type of fluid in the integration, i.e. $n=0,2,3,4$ and 6 , analytical solution can be found for all $n$ vales in complicated forms. For example, the simplest among these is dust case $(n=3)$ which has solution:

$$
\phi(t)= \pm \frac{2 \tau}{3 D \sqrt{\epsilon}}\left(\frac{2 k}{\kappa^{2}}-D e^{-t / \tau}\right)^{3 / 2}+\phi_{0},
$$

with additional rule that $k \geq 0$ and $\epsilon=1$ otherwise it is imaginary. In the next section, we will show how to obtain solution in NLS formulation for dust and radiation cases.

\footnotetext{
${ }^{2}$ We are not considering a cosmological constant but a dynamical scalar field and a barotropic fluid which together yield $w_{\text {eff }}=-1$.
} 


\section{Exponential expansion: Solutions solved with NLS formulation}

For exponential expansion, following Eqs. (11) and (15), we get

$$
u(x)=\dot{x}(t)=\exp (-n t / 2 \tau) .
$$

Integrating the above equation, hence parameters $x$ and $t$ scale as

$$
x(t)=-\frac{2 \tau}{n} e^{-n t / 2 \tau}+x_{0},
$$

where $x_{0}$ is an integration constant. The reverse is

$$
t(x)=-\frac{2 \tau}{n} \ln \left[(-n / 2 \tau)\left(x-x_{0}\right)\right]
$$

where the condition $x<x_{0}$ must be imposed. Now we can write wave function as

$$
u(x)=-\frac{n}{2 \tau}\left(x-x_{0}\right),
$$

which is a linear function. Using Eq. (22), hence the Eq. (13) reads

$$
P(t)=\frac{k n}{2} e^{(n-2) t / \tau}-\frac{\kappa^{2} n^{2} D}{12} .
$$

Here the Schrödinger kinetic energy term is

$$
T(t)=-\frac{k n}{2} e^{(n-2) t / \tau} .
$$

Expressing in Schrödinger formulation, these functions are written in term of $x$,

$$
\begin{aligned}
& P(x)=\frac{k n}{2}\left[-\frac{n}{2 \tau}\left(x-x_{0}\right)\right]^{-2(n-2) / n}-\frac{\kappa^{2} n^{2} D}{12}, \\
& T(x)=-\frac{k n}{2}\left[-\frac{n}{2 \tau}\left(x-x_{0}\right)\right]^{-2(n-2) / n}
\end{aligned}
$$

In order to obtain the scalar field potential $V(t)$, we use Eqs. (111), (12), (13) in Eq. (18), we finally obtain

$$
V(t)=\frac{3}{\kappa^{2} \tau^{2}}+\frac{2 k}{\kappa^{2}} e^{-2 t / \tau}+\left(\frac{n-6}{6}\right) D e^{-n t / \tau}
$$

which is checked by using Eq. (19) in standard formula (10). We use Eq. (32) in Eq. (17), then

$$
\psi(x)=\frac{ \pm 2}{\kappa \sqrt{n}} \times \int \sqrt{\frac{k n}{2 \epsilon}\left[\frac{-n}{2 \tau}\left(x-x_{0}\right)\right]^{-2(n-2) / n}-\frac{\kappa^{2} n^{2} D}{12 \epsilon}} \mathrm{d} x .
$$

We will integrate this equation in cases of $k=0$ and $k \neq 0$.

\subsection{The case $k=0$}

When $k=0$ and $D \neq 0$ integrating Eq. (35) and transforming $x$ to $t$ with Eq. (27) yields same result as Eq. (21) obtained by solving effective equation of state equation or by integrating from the Friedmann formulation. Real solution exists only when the scalar field is phantom. With the solution (21), the scalar field potential in term of $\phi$, reads

$$
V(\phi)=\frac{3}{\kappa^{2} \tau^{2}}+\left(\frac{n-6}{6}\right) \frac{3 n}{4 \tau^{2}}\left(\phi-\phi_{0}\right)^{2} .
$$




\subsection{The case $k \neq 0$}

When $k \neq 0$ and $D \neq 0$, the integral (35) can be integrated yielding complicated hypergeometric function even when $n$ is not specified. The case $n=0$ is excluded from our consideration by the reason mentioned in Sec 3 . For naturalness, we consider radiation $(n=4)$ and dust $(n=3)$.

\subsubsection{Radiation case}

Radiation fluid corresponds to $n=4$, the integral (35) becomes

$$
\psi(x)= \pm \frac{1}{\kappa} \int \sqrt{-\frac{k \tau}{\epsilon} \frac{1}{\left(x-x_{0}\right)}-\frac{4}{3} \frac{\kappa^{2} D}{\epsilon}} \mathrm{d} x .
$$

Here $x$ could be negative, $\epsilon$ can possibly be either \pm 1 . The solution in radiation case is

$$
\begin{aligned}
\psi(x)= & \pm \sqrt{\frac{1}{\epsilon}\left[-\frac{4}{3} D\left(x-x_{0}\right)^{2}-\frac{k \tau}{\kappa^{2}}\left(x-x_{0}\right)\right]} \\
& \pm \frac{k \tau}{4 \kappa^{2}} \sqrt{\frac{3}{D \epsilon}} \arctan \left\{\frac{\left[8 \kappa^{2} D\left(x-x_{0}\right) / 3 \epsilon\right]+k \tau / \epsilon}{[4 \kappa \sqrt{D} /(\epsilon \sqrt{3})] \sqrt{-\left[4 \kappa^{2} D\left(x-x_{0}\right)^{2} / 3\right]-k \tau\left(x-x_{0}\right)}}\right\}+\psi_{0},
\end{aligned}
$$

allowing only $\epsilon=1$ case for the solution to be real. Transforming $x$ scale to the $t$ scale using Eq. (27), the solution therefore reads

$$
\begin{aligned}
\phi(t)= & \pm \sqrt{\frac{1}{\epsilon}\left(-\frac{D \tau^{2}}{3} e^{-4 t / \tau}+\frac{k \tau^{2}}{2 \kappa^{2}} e^{-2 t / \tau}\right)} \\
& \pm \frac{k \tau}{4 \kappa^{2}} \sqrt{\frac{3}{D \epsilon}} \times \arctan \left\{\frac{-\left[4 \kappa^{2} D \tau /(3 \epsilon)\right] e^{-2 t / \tau}+k \tau / \epsilon}{[4 \kappa \sqrt{D} /(\epsilon \sqrt{3})] \sqrt{-\left(\kappa^{2} D \tau^{2} / 3\right) e^{-4 t / \tau}+\left(k \tau^{2} / 2\right) e^{-2 t / \tau}}}\right\}+\phi_{0}
\end{aligned}
$$

The solution above, when assuming $k=0$, reduces to the solution (21) when $n=4$, confirming the correctness of the result obtained.

\subsubsection{Dust case}

The integral (35) in the dust case $n=3$ reads

$$
\psi(x)= \pm \frac{2}{\kappa \sqrt{3}} \int \sqrt{\left(\frac{3}{2} \tau^{2}\right)^{1 / 3} \frac{k}{\epsilon} \frac{1}{\left(x-x_{0}\right)^{2 / 3}}-\frac{3}{4} \frac{\kappa^{2} D}{\epsilon}} \mathrm{d} x
$$

with solutions

$$
\psi(x)= \pm \sqrt{\frac{D}{\epsilon}}\left[\left(\frac{3 \tau^{2}}{2}\right)^{1 / 3} \frac{4 k}{3 \kappa^{2} D}-\left(x-x_{0}\right)^{2 / 3}\right]^{3 / 2}+\psi_{0} .
$$

With similar procedure to the radiation case, using (27), the solution is therefore,

$$
\phi(t)= \pm \frac{2 \tau}{3 D \sqrt{\epsilon}}\left(\frac{2 k}{\kappa^{2}}-D e^{-t / \tau}\right)^{3 / 2}+\phi_{0},
$$

which is the same as Eq. (25) derived from Friedmann formulation. This solution when assuming $k=0$ is exactly the same as the solution (21) when $n=3$ (dust fluid). This also confirms that our results from NLS formulation are correct. The NLS solution can solve the case when $k$ and $D$ are non-zero together without knowing $n$ value while the standard procedures in Sec. 4.2 can not unless assuming a particular value $n=0,2,3,4,6$. However, it must be noticed that one can not reduce the NLS solutions (39) and (42) to the $D=0$ case directly since there are mixed multiplication term of $n$ and $k$ in the solution and also the value of $n$ has already been put in. Hence setting $D=0$ in (39) and (42) can not be considered as a pure scalar field dominant case. 


\section{Exponential expansion: Analysis on effective equation of state coefficient}

The exponential expansion in our scenario is caused from mixed effect of fluids and spatial curvature. We discuss mixed effect on equation of state here. Definition of effective equation of state coefficient, $w_{\text {eff }}=\left(\rho_{\phi} w_{\phi}+\rho_{\gamma} w_{\gamma}\right) / \rho_{\text {tot }}$ together with Eqs. (44), (22) and (34) in context of exponential expansion becomes

$$
w_{\mathrm{eff}}=\frac{-1-\left(k \tau^{2} / 3\right) e^{-2 t / \tau}}{1+k \tau^{2} e^{-2 t / \tau}}
$$

which is infinite when

$$
t=\frac{\tau}{2} \ln \left(-k \tau^{2}\right)
$$

Infinity can possibly happen only when $k=-1$ because logarithm function forbids negative domain. In order to acquire exponential expansion in flat universe, one needs to have $w_{\text {eff }}=-1$, but this is not true when $k$ term is non-trivial. Therefore we can only express $w_{\phi}$ in term of $w_{\text {eff }}$ as

$$
w_{\phi}=\frac{\left[\left(3 k / \kappa^{2}\right) e^{-2 t / \tau}+3 /\left(\kappa^{2} \tau^{2}\right)\right] w_{\mathrm{eff}}-[(n-3) / 3] D e^{-n t / \tau}}{\left(3 k / \kappa^{2}\right) e^{-2 t / \tau}+3 /\left(\kappa^{2} \tau^{2}\right)-D e^{-n t / \tau}}
$$

for exponential expansion. The Eq. (43) does not depend on properties $(n)$ or amount $(D)$ of the barotropic fluid. It reduces to $w_{\text {eff }}=-1$ when $k=0$ as expected. Considering Eq. (45), if $D=0$ and $k=0$, it yields $w_{\phi}=w_{\text {eff }}$ while setting $D=0$ alone also gives the same result.

\section{Power-law expansion}

7.1 Bound value of $\phi(t)$ from effective equation of state for $k=0$ case

In power-law expanding universe, scale factor evolves with time as

$$
a(t)=t^{q}
$$

where $q>0$ is a constant. In flat $(k=0)$ universe, it is known that the power-law expansion, is attained when $-1<w_{\text {eff }}<-1 / 3$ where $q=2 /\left[3\left(1+w_{\text {eff }}\right)\right]$. The effective equation of state, (Eq. (1D) hence is a condition

$$
-\frac{n}{3} \rho_{\gamma}<\epsilon \dot{\phi}^{2}<\frac{2}{3}\left(\frac{\epsilon \dot{\phi}^{2}}{2}+V\right)+\left(\frac{2-n}{3}\right) \rho_{\gamma}
$$

i.e. $0<\epsilon \dot{\phi}^{2}+\rho_{\gamma} n / 3<(2 / 3) \rho_{\text {tot }}$. Both values of $\epsilon$ can be assigned and the power-law expansion is sustained as long as the condition is satisfied.

7.2 Solution solved from Friedmann formulation

If we directly consider Eq. (91), the solution for power-law expansion is an integration:

$$
\phi(t)= \pm \int \sqrt{\frac{1}{\epsilon}\left(\frac{2 q}{\kappa^{2} t^{2}}+\frac{2 k}{\kappa^{2} t^{2 q}}-\frac{n D}{3 t^{q n}}\right)} \mathrm{d} t .
$$




\subsubsection{Simplest case}

Simplest integration case is when $k=0$ and $D=0$. The solution of Eq. (48) is well known [24],

$$
\phi(t)= \pm \sqrt{\frac{2 q}{\epsilon \kappa^{2}}} \ln t+\phi_{0},
$$

provided that $q$ and $\epsilon$ have the same sign. Considering power-law inflation, the WMAP five-year combined analysis based on flat and scalar field domination assumption yields $q>60$ at more than 99 $\%$ of confident level otherwise excluded while $q \sim 120$ is at boundary of $68 \%$ confident level [9]. These results base on single field model which we can applied the above solution to. When assuming only $k=0$ with $D \neq 0$, the solution of Eq. (48) is

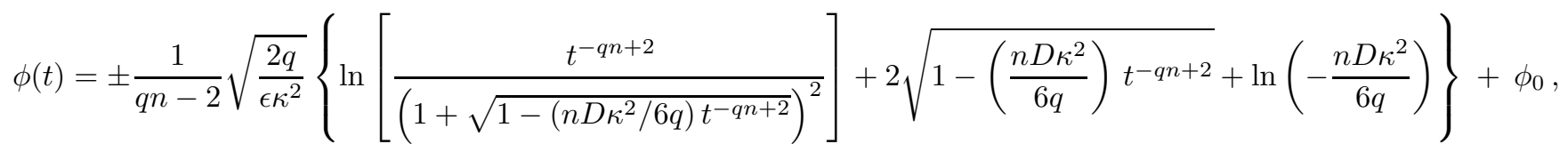

where, when $q=2 / n$, the field has infinite value. The last logarithmic term in the bracket is an integrating constant which is valid only when $q<0$. To attain power-law expansion, $q$ must be positive. Hence, this term is not defined for power-law expansion. We will see later that the NLS result does not have this problem. For the reverse case, $D=0, k \neq 0$, the solution is

$$
\phi(t)= \pm \frac{1}{q-1} \sqrt{\frac{2 q}{\epsilon \kappa^{2}}}\left\{\ln \left[\frac{t^{q-1}}{\sqrt{k / q}}\left(1+\sqrt{\left(\frac{k}{q}\right) t^{-2 q+2}+1}\right)\right]-\sqrt{\left(\frac{k}{q}\right) t^{-2 q+2}+1}\right\}+\phi_{0},
$$

which becomes infinite when $q=1$. The values of $q, k$ and $\epsilon$ must have the same sign in all terms of the solution otherwise becoming imaginary. Hence, for $q>0$, the condition for the solution to be valid is $k=1$ and $\epsilon=1$.

\subsubsection{The case of non-zero $k$ and non-zero $D$}

When considering non negligible value of both $k$ and $D$, the Eq. (48) can not be integrated analytically except when setting $n=2\left(w_{\gamma}=-1 / 3\right)$ which is not natural fluid. Hence it is not considered.

\section{Power-law expansion: Solutions solved with NLS formulation}

Power-law expansion cosmology in NLS-type formulation is presented and concluded in [23]. Important functions needed for evaluating the field exact solutions are

$$
\begin{aligned}
x & =\sigma(t)=-\frac{t^{-\beta}}{\beta}+x_{0}, \\
t(x) & =\frac{1}{\left[-\beta\left(x-x_{0}\right)\right]^{1 / \beta}}, \\
\epsilon \dot{\phi}(t)^{2} & =\frac{2 q}{\kappa^{2} t^{2}}+\frac{2 k}{\kappa^{2} t^{2 q}}-\frac{n D}{3 t^{q n}}, \\
P(x) & =\frac{2 q n}{(q n-2)^{2}} \frac{1}{\left(x-x_{0}\right)^{2}}+\frac{k n}{2}\left[\frac{-2}{(q n-2)\left(x-x_{0}\right)}\right]^{2 q(n-2) /(q n-2)}-\frac{\kappa^{2} n^{2} D}{12}, \\
V(t) & =\frac{q(3 q-1)}{\kappa^{2} t^{2}}+\frac{2 k}{\kappa^{2} t^{2 q}}+\left(\frac{n-6}{6}\right) \frac{D}{t^{q n}} .
\end{aligned}
$$

We use Eq. (55) in Eq. (17), then

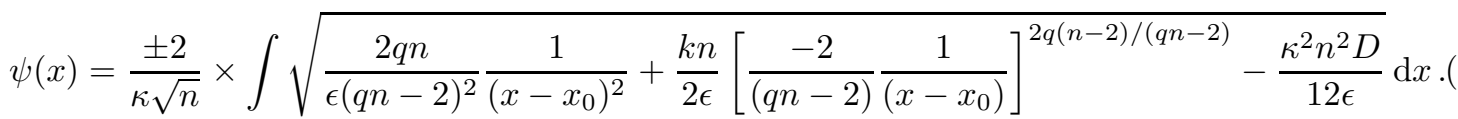


We consider the solution in cases of $k=0$ and $k \neq 0$. Recall that setting $D=0$ can not be considered as an absence of barotropic fluid due to existence of $n$ in the other terms.

8.1 The case $k=0$

Solution to the integral (57) for $k=0$ case is

$$
\begin{aligned}
\psi(x)= & \pm \sqrt{\frac{8 q}{\epsilon \kappa^{2}(q n-2)^{2}}} \times \\
& \left\{-\sqrt{1-\left[\frac{\kappa^{2} D n(q n-2)^{2}}{24 q}\left(x-x_{0}\right)^{2}\right]}+\ln \left[\frac{1+\sqrt{1-\left[\kappa^{2} D n(q n-2)^{2} / 24 q\right]\left(x-x_{0}\right)^{2}}}{\left(x-x_{0}\right)} \frac{4 q n}{\epsilon(q n-2)^{2}}\right]\right\} .
\end{aligned}
$$

Transforming to the $t$ variable using Eq. (52), we obtain,

$\phi(t)= \pm \frac{1}{q n-2} \sqrt{\frac{2 q}{\epsilon \kappa^{2}}}\left\{\ln \left[\frac{t^{-q n+2}}{\left(1+\sqrt{1-\left(n D \kappa^{2} / 6 q\right) t^{-q n+2}}\right)^{2}}\right]+2 \sqrt{1-\left(\frac{n D \kappa^{2}}{6 q}\right) t^{-q n+2}}+\ln \left(\frac{q n-2}{2 q n}\right)^{2}\right\}+\phi_{0}$.

This solution differs from the solution (50) only the last logarithmic term in the bracket which is only an integrating constant. When $q=2 / n$ or $n=0$, the field has infinite value. The last logarithmic term does not restrict the sign of $q$. Only $q$ and $\epsilon$ must have the same sign for the solution to be real.

\subsection{The case $k \neq 0$}

In case of non-zero $k$ and non-zero $D$, the integral (57) can not be integrated analytically even when assuming each $n$ value except when $n=2$ which is not natural fluid.

\section{Power-law expansion: Analysis on effective equation of state coefficient}

Similar to the analysis in Sec. 6, mixed effect of the two fluids and spatial curvature results in power-law expansion. The coefficient $w_{\text {eff }}$, with Eqs. (44), (54) and (56), reads

$$
w_{\mathrm{eff}}=\frac{\left(-3 q^{2}+2 q\right) t^{2 q-2}-k}{3 q^{2} t^{2 q-2}+3 k},
$$

which becomes infinity if

$$
t=\left(\frac{-k}{q^{2}}\right)^{1 /(2 q-2)}
$$

We can also express $w_{\phi}$ in term of $w_{\text {eff }}$ as

$$
w_{\phi}=\frac{\left[\left(3 q^{2} / \kappa^{2}\right) t^{-2}+\left(3 k / \kappa^{2}\right) t^{-2 q}\right] w_{\mathrm{eff}}-[(n-3) / 3] D t^{-q n}}{\left(3 q^{2} / \kappa^{2}\right) t^{-2}+\left(3 k / \kappa^{2}\right) t^{-2 q}-D t^{-q n}},
$$

for power-law expansion. The Eq. (62), when $D=0$ and $k=0$, yields $w_{\phi}=w_{\text {eff }}$ as expected. Similar to the case of exponential expansion, setting $D=0$ alone also yields $w_{\phi}=w_{\text {eff }}$. In flat universe, power-law expansion happens when $w_{\text {eff }}$ lies in an interval $(-1,-1 / 3)$. But in $k \neq 0$ universe, it is no longer true. Considering flat universe, setting $k=0$ in Eq. (60) yields $q=2 /\left[3\left(1+w_{\text {eff }}\right)\right]$. The condition $-1<w_{\text {eff }}<-1 / 3$ therefore corresponds to $q>0$ as known. The condition also yields

$$
-1-\left(1+w_{\gamma}\right) \frac{\rho_{\gamma}}{\rho_{\phi}}<w_{\phi}<-\frac{1}{3}-\left(\frac{1}{3}+w_{\gamma}\right) \frac{\rho_{\gamma}}{\rho_{\phi}} .
$$


If there is more non-negligible radiation fluid (with $w_{\gamma}=1 / 3$ ), it is noticed that the interval shifts to the more left. For example, setting $\rho_{\gamma}=0.1 \rho_{\phi}$, the interval shifts to about $-1.133<w_{\phi}<-0.4$. If we assume more realistic situation when dust (dark matter and other matter elements) is presented. The dust density and dark energy is about $28 \%$ and $72 \%$ of total density, therefore $\rho_{\gamma} \simeq(28 / 72) \rho_{\phi} \simeq$ $0.389 \rho_{\phi}$, the interval is $-1.389<w_{\phi}<-0.463$ which covers valid range of recent observational data, assuming dynamical $w$ with flat universe, $-1.38<w_{\phi, 0}<-0.86$ at $95 \%$ confident level [9].

\section{Conclusions}

This letter reports and demonstrates a method of solving for canonical scalar field exact solution in a non-flat FLRW universe with barotropic fluid using non-linear Schrödinger (NLS)-type formulation in comparison with the method in the standard Friedmann framework. We consider phantom and non-phantom scalar field cases with exponential and power-law accelerating expansion. We evaluate all NLS quantities needed to find the solution, e.g. non-normalizable wave function and Schrödinger potential. Our process is reverse to a problem solving in quantum mechanics that the wave function is expressed first by the expansion function, $a(t)$ before evaluating the Schrödinger potential based on a known expansion function. In NLS formulation the total energy $E$ is negative. We do an analysis on effective equation of state to both cases of expansion. We expresses $w_{\text {eff }}$ in term of $q$ and $k$. In a flat universe, in order to have power-law expansion, the interval $(-1,-1 / 3)$ of the $w_{\phi}$, is shifted leftward to more negative if more barotropic fluid density is presented.

Within framework of the standard Friedmann formulation, we obtained exact solution in various cases. Later we solved the problem using NLS formulation, in which the wave function is equivalent to the scalar field exact solution. NLS method is restricted by the fact that its scalar field solution is valid only when the barotropic fluid density is presented. Setting $D=0$ does not imply the absence of barotropic fluid because the barotropic fluid parameter $n$ still appears in the other terms of the Schrödinger potential. Therefore NLS formulation can not be applied to situation when the scalar field is dominant and $D \sim 0$. Hence it is more suitable for a system of dark energy and dust dark matter fluid. This is a disadvantage point of the NLS formulation. Transforming from standard Friedmann formulation to NLS formulation makes $n$ appear in all terms of the integrand and also changes fluid density term $D$ from time-dependent term to a constant $E$. Hence the number of $x$ (or equivalently $t$ )-dependent terms is reduced by one. This is a good aspect of the NLS. In both Friedmann formulation and NLS formulation, the solutions when $k \neq 0$ and $D \neq 0$ are difficult or might be impossible to solve unless assuming values of $q$ and $n$. Hence reduction number of $x$-dependent term helps simplifying the integration. There are also other good aspects of NLS formulation. Firstly, in the case of exponential expansion with NLS formulation, the solution when $k \neq 0$ and $D \neq 0$ can be obtained without assuming $n$ value while $n=0,2,3,4,6$ must be given if working within Friedmann formulation. Secondly, for power-law expansion with $k=0$, the result (59) obtained from NLS formulation has integrating constant that does not restrict $q$ value while (50) obtained from Friedmann formulation needs $q<0$ which violates power-law expansion condition $(q>0)$. For power-law expansion, the most difficult case is when $k \neq 0$ with $D \neq 0$. In both formulations, the integral can not be integrated unless assuming $n=2$ (equivalent to $w_{\gamma}=-1 / 3$ ) which is not a physical fluid. We introduce here alternative method to obtain scalar field exact solution with advantage over and disadvantage to standard Friedmann formulation. The NLS formulation could render more interesting techniques for scalar field cosmology.

Acknowledgements B. G. is a TRF Research Scholar of the Thailand Research Fund. B. G. gives a special thank for hospitalities, supports during his research visits and for seminal invitations, to Yupeng Yan, Prapan Manyum and Chinorat Kobdaj at Suranaree University of Technology, to Department of Physics of Ubon Rajathanee University and to the organizers of the Forth Aegean Summer School on Black Holes \& the First Annual School of the EU Network UniverseNet-The Origin of the Universe in Mytilini, Island of Lesvos, Greece where this work was partially completed.

\section{References}

1. Scranton, R., et al.: [SDSS Collaboration], Physical evidence for dark energy. arXiv: astro-ph/0307335 
2. Riess, A. G., et al.: [Supernova Search Team Collaboration], Observational evidence from supernovae for an accelerating universe and a cosmological constant. Astron. J. 116, 1009 (1998); Perlmutter, S., et al.: [Supernova Cosmology Project Collaboration], Measurements of $\Omega$ and $\Lambda$ from 42 high-redshift supernovae. Astrophys. J. 517, 565 (1999); Riess, A. G., Peculiar velocities from type Ia supernovae. arXiv: astro-ph/9908237 Goldhaber, G., et al.: [The Supernova Cosmology Project Collaboration], Timescale stretch parameterization of type Ia supernova B-band light curves. arXiv: astro-ph/0104382, Tonry, J. L., et al.: [Supernova Search Team Collaboration], Cosmological results from high- $z$ supernovae. Astrophys. J. 594, 1 (2003).

3. Riess, A. G., et al.: [Supernova Search Team Collaboration], Type Ia supernova discoveries at $z>1$ from the Hubble Space Telescope: evidence for past deceleration and constraints on dark energy evolution. Astrophys. J. 607, 665 (2004); Riess, A. G., et al., New Hubble Space Telescope discoveries of type Ia supernovae at $z>1$ : narrowing constraints on the early behavior of dark energy. Astrophys. J. 659, 98 (2007).

4. Astier, P., et al.: [SNLS Collaboration], The Supernova Legacy Survey: measurement of $\Omega_{M}, \Omega_{\Lambda}$ and $w$ from the first year data set. Astron. Astrophys. 447, 31 (2006).

5. Kazanas, D., Dynamics of the universe and spontaneous symmetry breaking. Astrophys. J. 241, L59 (1980); Starobinsky, A. A., A new type of isotropic cosmological models without singularity. Phys. Lett. B 91, 99 (1980); Guth, A. H., Inflationary universe: a possible solution to the horizon and flatness problems. Phys. Rev. D 23, 347 (1981); Sato, K., First-order phase transition of a vacuum and the expansion of the universe. Mon. Not. Roy. Astro. Soc. 195, 467 (1981); Albrecht, A. and Steinhardt, P. J., Cosmology for Grand Unified Theories with radiatively induced symmetry breaking. Phys. Rev. Lett. 48, 1220 (1982); Linde, A. D., A new inflationary universe scenario: a possible solution of the horizon, flatness, homogeneity, isotropy and primordial monopole problems. Phys. Lett. B 108, (1982) 389.

6. Spergel, D. N., et al.: [WMAP Collaboration], Wilkinson Microwave Anisotropy Probe (WMAP) three year results: implications for cosmology. Astrophys. J. Suppl. 170, 377 (2007).

7. Hinshaw, G., et al.: [WMAP Collaboration], Five-Year Wilkinson Microwave Anisotropy Probe (WMAP) observations: data processing, sky maps, \& basic results. arXiv:0803.0732 [astro-ph].

8. Dunkley, J., et al.: [WMAP Collaboration], Five-Year Wilkinson Microwave Anisotropy Probe (WMAP) observations: likelihoods and parameters from the WMAP data. arXiv:0803.0586 [astro-ph].

9. Komatsu, E., et al.: [WMAP Collaboration], Five-Year Wilkinson Microwave Anisotropy Probe (WMAP) observations: cosmological interpretation. arXiv:0803.0547] [astro-ph].

10. Padmanabhan, T., Dark energy: the cosmological challenge of the millennium. Curr. Sci. 88, 1057 (2005); Copeland, E. J., Sami, M. and Tsujikawa, S., Dynamics of dark energy. Int. J. Mod. Phys. D 15, 1753 (2006); Padmanabhan, T., Dark energy: mystery of the millennium. AIP Conf. Proc. 861, 179 (2006).

11. Caldwell, R. R., A phantom menace? Phys. Lett. B 545, 23 (2002); Gibbons, G. W., Phantom matter and the cosmological constant. arXiv:hep-th/0302199 Nojiri, S., and Odintsov, S. D., Quantum deSitter cosmology and phantom matter. Phys. Lett. B 562, 147 (2003).

12. Melchiorri, A., Mersini-Houghton, L., Odman, C. J., and Trodden, M., The state of the dark energy equation of state. Phys. Rev. D 68, 043509 (2003); Corasaniti, P. S., Kunz, M., Parkinson, D., Copeland, E. J. and Bassett, B. A., The foundations of observing dark energy dynamics with the Wilkinson Microwave Anisotropy Probe. Phys. Rev. D 70, 083006 (2004); Alam, U., Sahni, V., Saini, T. D. and Starobinsky, A. A., Is there supernova evidence for dark energy metamorphosis? Mon. Not. Roy. Astron. Soc. 354, 275 (2004).

13. Wood-Vasey, W. M., et al.: [ESSENCE Collaboration], Observational constraints on the nature of the dark energy: first cosmological results from the ESSENCE supernova survey. Astrophys. J. 666, 694 (2007).

14. Percival, W. J., Cole, S., Eisenstein, D. J., Nichol, R. C., Peacock, J. A., Pope, A. C., and Szalay, A. S., Measuring the Baryon Acoustic Oscillation scale using the SDSS and 2dFGRS. Mon. Not. Roy. Astron. Soc. 381, 1053 (2007).

15. Steigman, G., Primordial nucleosynthesis in the precision cosmology era. Ann. Rev. Nucl. Part. Sci. 57, 463 (2007).

16. Wright, E. L., Constraints on dark energy from supernovae, gamma ray bursts, acoustic oscillations, nucleosynthesis and large scale structure and the Hubble constant. Astrophys. J. 664, 633 (2007).

17. Caldwell, R. R., Kamionkowski, M. and Weinberg, N. N., Phantom energy and cosmic doomsday. Phys. Rev. Lett. 91, 071301 (2003); Nesseris, S. and Perivolaropoulos, L., The fate of bound systems in phantom and quintessence cosmologies. Phys. Rev. D 70, 123529 (2004); Hao, J. g. and Li, X. z., An attractor solution of phantom field. Phys. Rev. D 67, 107303 (2003); Li, X. z. and Hao, J. g., O $(N)$ phantom, a way to implement $w<-1$. Phys. Rev. D 69, 107303 (2004); Singh, P., Sami, M. and Dadhich, N., Cosmological dynamics of phantom field. Phys. Rev. D 68, 023522 (2003); Hao, J. g. and Li, X. z., Phantom cosmic doomsday: a tale of two attractors. Phys. Rev. D 70, 043529 (2004); Sami, M. and Toporensky, A., Phantom field and the fate of universe. Mod. Phys. Lett. A 19, 1509 (2004); Nojiri, S., Odintsov, S. D., and Tsujikawa, S., Properties of singularities in (phantom) dark energy universe. Phys. Rev. D 71063004 (2005); Gumjudpai, B., Naskar, T., Sami, M. and Tsujikawa, S., Coupled dark energy: towards a general description of the dynamics. J. Cosmol. Astropart. Phys. 0506, 007 (2005); Urena-Lopez, L. A., Scalar phantom energy as a cosmological dynamical system. J. Cosmol. Astropart. Phys. 0509, 013 (2005).

18. Nojiri, S., Odintsov, S. D. and Sasaki, M., Gauss-Bonnet dark energy. Phys. Rev. D 71, 123509 (2005); Sami, M., Toporensky, A., Tretjakov, P. V. and Tsujikawa, S., The fate of (phantom) dark energy universe with string curvature corrections. Phys. Lett. B 619, 193 (2005); Calcagni, G., Tsujikawa, S. and Sami, M., Dark energy and cosmological solutions in second-order string gravity. Class. Quant. Grav. 22, 3977 (2005); Wei, H. and Cai, R. G., Cosmological evolution of hessence dark energy and avoidance of big rip. Phys. Rev. D 72, 123507 (2005); Leith, B. M. and Neupane, I. P., Gauss-Bonnet cosmologies: crossing the phantom divide and the transition from matter dominance to dark energy. J. Cosmol. Astropart. Phys. 0705, 019 (2007); Samart, D. and Gumjudpai, B., Phantom field dynamics in loop quantum cosmology. Phys. Rev. 
D 76, 043514 (2007); Naskar, T. and Ward, J., Phys. Rev. D 76, 063514 (2007); Gumjudpai, B., Coupled phantom field in loop quantum cosmology. Thai J. Phys. Series 3: Proc. of the SIAM Phys. Cong. (2007), arXiv:0706.3467 [gr-qc].

19. Hawkins, R. M. and Lidsey, J. E., The Ermakov-Pinney equation in scalar field cosmologies. Phys. Rev. D 66, 023523 (2002); Williams, F. L. and Kevrekidis, P. G., On (2+1)-dimensional Friedmann-Robertson-Walker universes: an Ermakov-Pinney equation approach. Class. Quant. Grav. 20, L177 (2003); Lidsey, J. E., Cosmic dynamics of Bose-Einstein condensates. Class. Quant. Grav. 21, 777 (2004); Williams, F. L., Kevrekidis, P. G., Christodoulakis, T., Helias, C., Papadopoulos, G. O. and Grammenos, T., On 3+1 dimensional scalar field cosmologies. Trends in Gen. Rel. and Quant. Cosmol., 37, Nova Science Pub. (2006); Kamenshchik, A. Luzzi, M. and Venturi, G., Remarks on the method of comparison equations (generalized WKB method) and the generalized Ermakov-Pinney equation. arXiv: math-ph/0506017

20. Williams, F. L., Einstein field equations: an alternate approach towards exact solutions for an FRW universe. Int. J. Mod. Phys. A 20, 2481 (2005)

21. D'Ambroise, J. and Williams, F. L., A non-linear Schrödinger type formulation of FLRW scalar field cosmology. Int. J. Pure Appl. Math. 34, 117 (2007).

22. D'Ambroise, J., A Schrödinger type formulation of Bianchi I scalar field cosmology. Int. J. Pure Appl. Math. 3, 417 (2008) arXiv:0711.3916 [hep-th].

23. Gumjudpai, B., Power-law expansion cosmology in Schrödinger-type formulation. arXiv: 0708.3674 [gr-qc].

24. Lucchin, F. and Matarrese, S., Power-law inflation. Phys. Rev. D 32, 1316 (1985). 